\title{
Analysis and Design of Three Dimensional Free Space Optoelectronic Switching Network
}

\author{
Yun-Parn Lee ${ }^{1}$, Jih-Huah $\mathrm{Wu}^{2}$ \\ ${ }^{1}$ Department of Electronic Engineering, Ming Chuan University, Taoyuan, Taiwan, ROC \\ ${ }^{2}$ Department of Biomedical Engineering, Ming Chuan University, Taoyuan, Taiwan, ROC \\ \{yplee@mail.mcu.edu.tw,wujh@mail.mcu.edu.tw \}
}

\begin{abstract}
Many research papers on optical interconnects have been published over the last three decades, however, some models are oversimplified and overlooked important design considerations. These include the timing synchronization of the VCSEL sources; detailed calculations of the free space optoelectronic interconnect network, theoretical analysis of computer generated hologram on optical interconnect system. An example of detailed analysis are presenting here for devising optoelectronic interconnect switching network based on a banyan switch. In order to expand the work into a large optoelectronic switching arrays, more careful considerations may be necessary from system perspective..

Keywords: Optoelectronic Banyan Switch, Time Synchronization, VCSEL Arrays, Temperature in Optoelectronics Interconnect.
\end{abstract}

\section{Introduction}

Early researches indicated that the integrated circuits have begun approaches the fundamental limits [1]. Since the electrical interconnection is treated as a uniform RLC transmission line, the coupling capacitance and on-chip inductance may cause addition delay and crosstalk, from timing diagram of electronics digital switching, because of the parasitic capacitance, voltage can not change instantaneously, in the following diagram Fig 1, this diagram defines the amount of time needed for logic signal change [2], this is called the propagation time delay $t_{P}$. We can express in the following equation [2], where this expression, the speed of logic switching depends on the value of capacitance $\mathrm{C}$ and the dimensions of the NMOS/PMOS transistor's width $W$ and length $L$.

$$
t_{P} \cong \frac{1.7 C}{k_{n}^{\prime} \frac{W}{L} V_{D D}}
$$

Many researchers have proposed holographic interconnections for free space interconnection; most of researches focus on Fresnel lens optical interconnects [3], other researchers proposed free space holographic interconnects [4][5][6][7]. M. Haurylau and G.Chen [8][9] indicated intra-chip optical interconnects have the potentials of outperform the electrical wires, they also perform analysis of delay as a function of interconnect width, and excellent comparison of delays, power of electrical and optical interconnects. Optical switching interconnect system time delay can be expressed in Fig 2, where in the equations, $\boldsymbol{t}_{\boldsymbol{s}}$ stands for the rise time of optical sources, $\boldsymbol{t}_{\boldsymbol{p}}$ represent the response time of optical detectors, and $\boldsymbol{t}_{\boldsymbol{e}}$ represent the response time of electronics cirtuits.

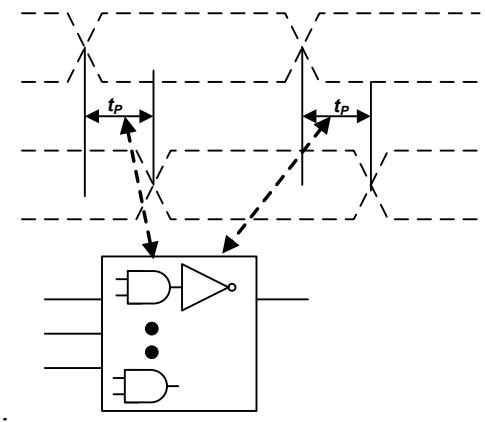

Fig. 1. Logic signal change waveform for electronics logic switching circuit

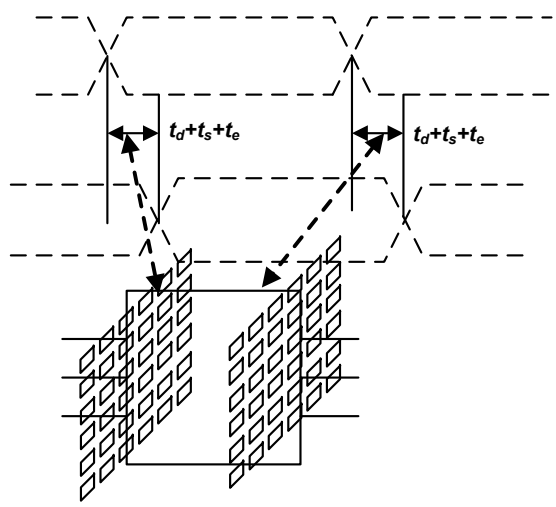

Fig 2. Logic signal change waveform for Optical Switching circuits.

\section{Design Analysis of Optoelectronic Banyan Switch Network}

Recent advances in high performance switching technologies [10][11] proves that Banyan switching network is one of the best choice among all different technologies. N.Andriollli [12] proposed using semiconductor optical amplifier (SOA) based photonic processing modules to implement the Banyan switching network, at earlier time. G.Li and S. Esener [13], F.E. Kiamilev [6] and G. Marsden [14] proposed similar approaches using free-space optoelectronics to implement the Banyan Switching network, however, the detailed derivations of their technical approaches were missing [4][7].

In Fig. 3 shows the architecture of optical crossbar switching interconnection network [13]. The optical crossbar 
switching system forms the key components of modern optical switching network. In previous work, N.Andriollli [12] has performed preliminary design of $2 \times 2$ optoelectronic Banyan switch, each block of their optoelectronic Banyan switch is a clock driven Moore state machine. We are not going to redraw the $2 \times 2$ switching circuit here [14]; rather, the switching circuit can be shown as in Fig. 4 and Fig. 5.

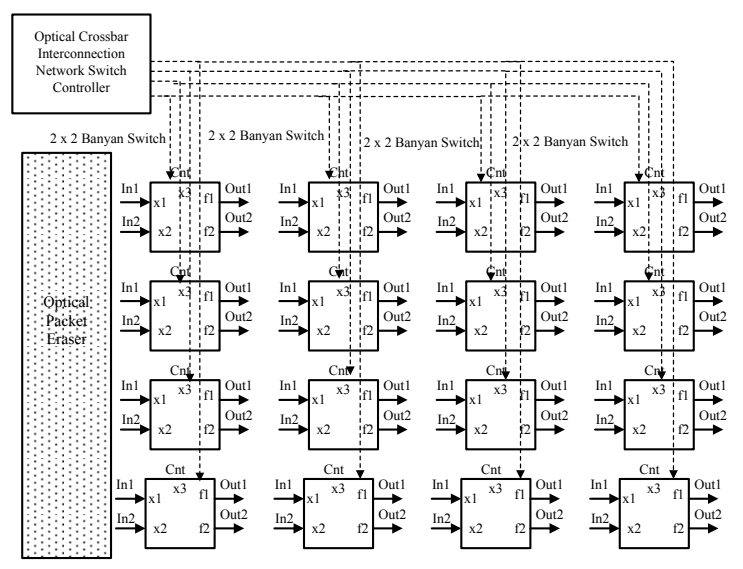

Fig 3. Architecture of an Optical Crossbar Switching Interconnection Network

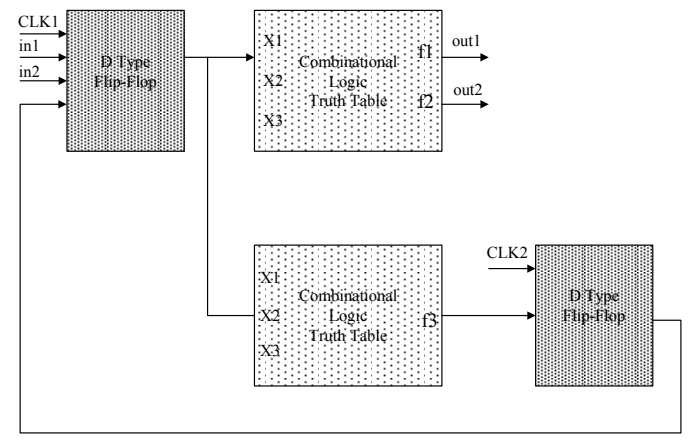

Fig 4. Architecture of 2x2 Optoelectronic Banyan Switch

\begin{tabular}{lll|llll|ll}
$x_{1}$ & $x_{2}$ & $x_{3}$ & $f_{1}$ & $f_{1}(z)$ & $f_{2}$ & $f_{2}(z)$ & $f_{3}$ & $f_{3}(z)$ \\
\hline 0 & 0 & 0 & 0 & +1 & 1 & -1 & 0 & +1 \\
0 & 0 & 1 & 1 & -1 & 0 & +1 & 0 & +1 \\
0 & 1 & 0 & 0 & +1 & 1 & -1 & 1 & -1 \\
0 & 1 & 1 & 1 & -1 & 0 & +1 & 1 & -1 \\
1 & 0 & 0 & 0 & +1 & 1 & -1 & 0 & +1 \\
1 & 0 & 1 & 1 & -1 & 0 & +1 & 0 & +1 \\
1 & 1 & 0 & 0 & +1 & 1 & -1 & 1 & -1 \\
1 & 1 & 1 & 1 & -1 & 0 & +1 & 1 & -1
\end{tabular}

Fig 5. Truth Table of Combinational Logics in Fig. 4.

An optical system is a linear mapping function such as matrix multiplication, correlation or convolution of matrixes. However a truth-table mapping is a nonlinear mapping, in the earlier works [3][6], we show how to convert a nonlinear truth-table mapping into a linear relationship. Therefore, the calculations of spectral expansion matrix of above combinatorial logic of a Banyan switch network are shown as following:

$$
\begin{aligned}
& R=T^{*} Z \\
& =\frac{1}{8}\left[\begin{array}{rrrrrrrr}
1 & 1 & 1 & 1 & 1 & 1 & 1 & 1 \\
1 & -1 & 1 & -1 & 1 & -1 & 1 & -1 \\
1 & 1 & -1 & -1 & 1 & 1 & -1 & -1 \\
1 & -1 & -1 & 1 & 1 & -1 & -1 & 1 \\
1 & 1 & 1 & 1 & -1 & -1 & -1 & -1 \\
1 & -1 & 1 & -1 & -1 & 1 & -1 & 1 \\
1 & 1 & -1 & -1 & -1 & -1 & 1 & 1 \\
1 & -1 & -1 & 1 & -1 & 1 & 1 & -1
\end{array}\right]\left[\begin{array}{cccc}
1 & -1 & 1 \\
-1 & 1 & 1 \\
1 & -1 & -1 \\
-1 & 1 & -1 \\
1 & -1 & 1 \\
-1 & 1 & 1 \\
1 & -1 & -1 \\
-1 & 1 & -1
\end{array}\right] \\
& =\frac{1}{8}\left[\begin{array}{ccc}
0 & 0 & 0 \\
8 & -8 & 0 \\
0 & 0 & 0 \\
0 & 0 & 0 \\
0 & 0 & 0 \\
0 & 0 & 0 \\
0 & 0 & 0 \\
0 & 0 & 0
\end{array}\right]=\left[\begin{array}{lll}
R_{1}(z) & R_{2}(z) & R_{3}(z)
\end{array}\right]
\end{aligned}
$$

Fig. 6 shows the addition of two separated components of $D_{++}, D_{--}$and $D_{+-}, D_{-+}$, go through a comparator circuit to get the final truth table outputs. This comparator circuit is an improved version of the previous circuits. The inputs of the truth table go through the Boolean basis operation as shown in Fig. 7. demonstrate the simulation result of the truth-table Boolean logic switching.

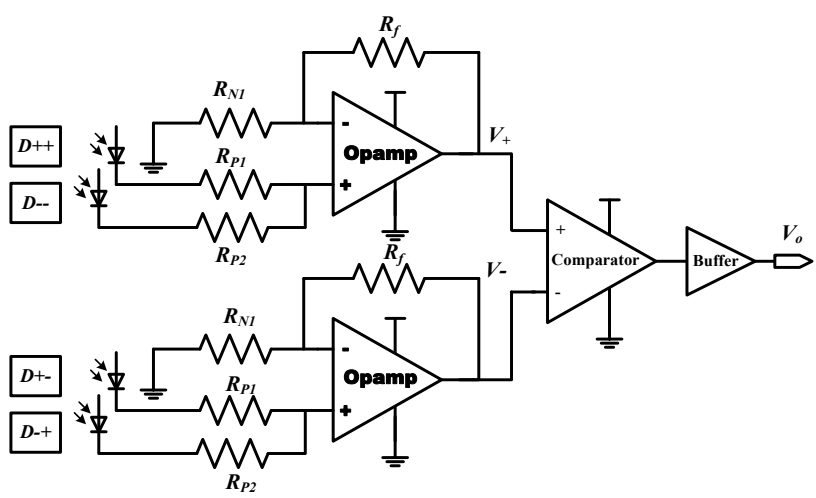

Fig 6. Detector Arrays and Comparators

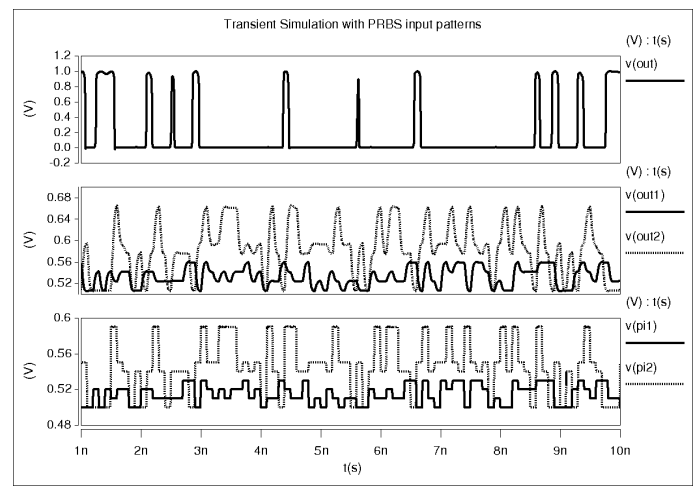

Fig 7. Optical Receiver Simulation Results 


\section{Design Considerations of VCSEL Arrays and Photodector Arrays}

\section{A. Timing Synchronization of VCSEL Arrays}

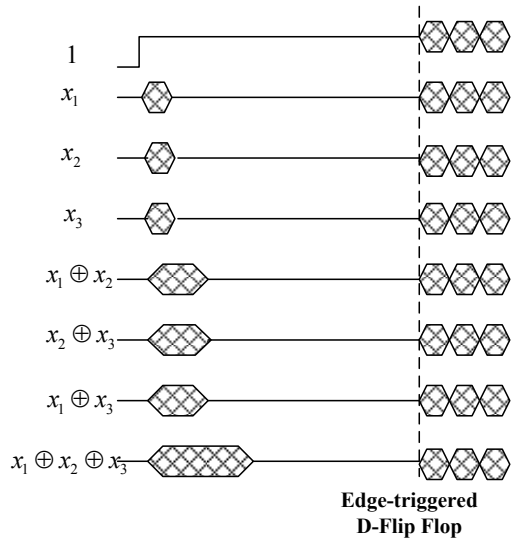

Fig. 8 Time Synchronization of VCSEL Arrays

From Fig. 5, we show a circuit to implement a three variables truth table mapping, therefore, we understand that different circuits to implement the Boolean basic vectors has different time delays as shown in Fig. 8 . Signals have to go through different signal paths. We need a synchronization mechanism to turn on/off the VCSEL arrays at the same time. Such time synchronization can make sure that the correct switching times of truth-table outputs in the optoelectronic system.

\section{B. Two Different Methods for the Optoelectronic System Design}
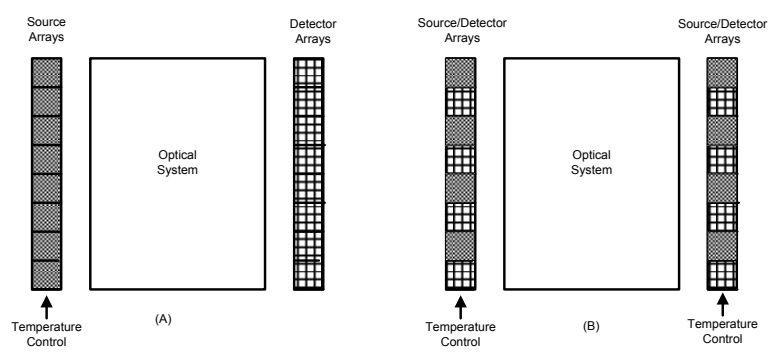

Fig 9. Two different methods for the optoelectronic system design in optical interconnects (A) Source Detector arrays in the different planes (B) Source Detector arrays in the same plane

There are two different methods for optoelectronics system design in optical interconnects. The first method is to separate the sources and detectors in two different planes [1516]. The second method is to integrate sources and detectors in the same plane [19-20], this is illustrated in Fig. 9. The major advantage of the first method can reduce the alignment error of the optoelectronic system. Since VCSELs and detectors are two types of different material [19-20], it is easier to design temperature control circuits for two types of material in two separated planes. If we integrate the sources and detectors in the same plane, this may cause extreme difficulty in designing temperature control circuits. In addition, the second method will have serious issues on alignment errors [17][18].

\section{Temperature Issues In Optoelectronic Interconnect System Design}

To the best of our knowledge, John .A. Neff was the first to suggest thermal considerations of free space optical interconnects using VCSEL based smart pixels and made significant contributions [21]. In free space optical intercoconnects, since the high temperature rising become an critical issue, there is less problem if we are only considering one or two VCSELs, however, for a large array of $256 \times 256$ matrix type of VCSELs, this will consumed large power.

In Figure 10, we show a 8 x 8 VCSEL arrays. Figure 11 shows an actual implementation diagram of VCSEL arrays in AIN substrate [23]. One of the key undersirable functions of VCSEL is the compilation of heat inside the laser cavity. Self generating high heat of VCSEL can accumulate excessive heat source, which limit the performance of VCSEL arrays, mainly due to it's internal high current density build up in the doped Bragg reflectors (DBRS). The increasing in temperature causes the increase the threshold current density, plus reduce the output power, and shift frequency to longer wavelength, in addition, it may reduce its lifetime. D. Botez [24] and S.Fu [25] indicate the steady state thermal rate equation inside cavity of VCSELs as following:

$$
\rho_{m} C_{P} \frac{\partial T}{\partial t}=\nabla \cdot\left(k_{T} \Delta T\right)+\rho_{T}
$$

Where $\rho_{m}$ is the mass density, $C_{P}$ is the heat capacity, $k_{T}$ is the thermal conductivity of the laser cavity, $\rho_{T}$ is the power density. D. Botez [24], S.Fu [25] and N.Bertra [26] did an excellent work on single VCSEL thermal analysis. They tried to extend the work through arrays of VCSEL using finite difference method and finite element method, however, the heat distribution on VCSELs array was difficult to estimate through those numerical methods.

We are curently working temperature estimation of evenly distributed two dimensional VCSELs array and temperature control circuits.

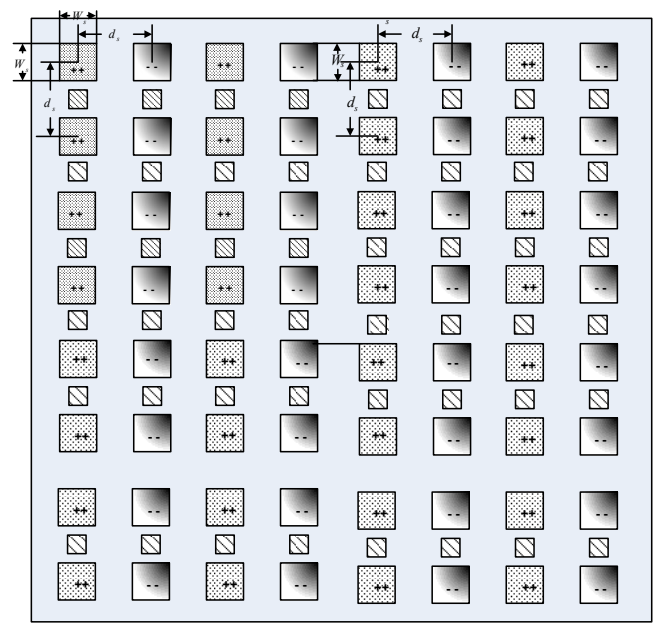

Figure 10. A $8 \times 8$ VCSELs array temperature estimation [4] 


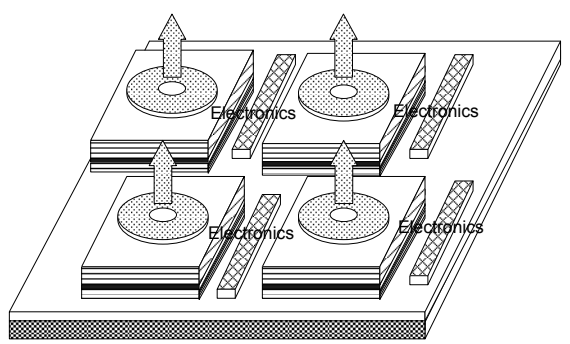

Figure 11 VCSEL arrays in AIN substrate [23]

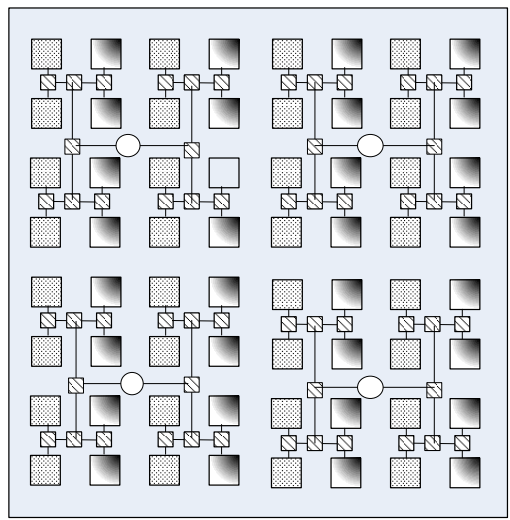

Figure 12. A 8 x 8 VCSELs array based on UCSD's work [5]

\section{Conclusions and Acknowledgement}

In previous works [5-6], authors did not show the detail calculations on how to implement the digital interconnect in optoelectronic interconnect networks. We showed [4][7]the calculations and methods to implement the Banyan switching network in optoelectronic implementations.

We also provide a solution to synchronize the VCSEL source arrays in time domain.

Temperature control becomes a critical issue of large VCSEL source arrays, the previous methods can not provide such calculations. We are currently working on a possible solution to this problems.

Finally, Authors wish to thank Dr. Yulai Zhang and Professor C.K. Cheng to provide the simulation result in Figure 7.

\section{References}

[1] Shigeru Kawai, Handbook of Optical Interconnects, CRC Press Taylor \& Francis Group,2005

[2] S. Brown, Z. Vranesic, Fundamentals of Digital Logic with Verilog Design, $2^{\text {nd }}$ Editition, Chapter 3, 2008, McGraw-Hill Inc.

[3] W.E. Wu, A. Bergman, A.R. Johnston, C. C. Guest, S.C.Esener, P.K.L. $\mathrm{Yu}$, M.R. Feldman, S.H.Lee, "Implementation of Optica Interconnections for VLSI", IEEE Transactions on Electron Devices, Vol. ED-34, No. 3, pp.706-714, March 1987

[4] Y.P.T.Lee,"Means and method for implementation of a two-dimensional truth-table look-up holographic process," U.S. Patent 4892370, Jan. 9, 1990.

[5] A.V.Krishnamoorthy, G. C. Mardsen, J.E. Ford and S.C. Esener,"Dualscale topology optoelectronic matrix algebraic processing system,"U.S Patent 5321639, June 14, 1994.

[6] Fouad E. Kiamilev, P. Marchand, A.V. Krishnamoorthy, S.C. Esener and S.H. Lee, "Performance Comparison Between Optoelectrnic and VLSI
Multistage Interconnection Networks," Journal of Lightwave Technology, Vol. 9, No. 12, pp. 1674-1692, December 1991.

[7] Yun-Parn Lee, Yulei Zhang, "Performance Comparison and Overview of Different Approaches for VLSI Optoelectronic Interconnects", IEEE/OSA J. Opt. Commun. Networks, Vol 2, No. 4, pp 206-220, April 2010.

[8] M. Haurylau, C. Chen, Hui Chen, Jidong Zhang, N.A. Nelson, D.H. Albonesi, E.G. Friedman, P.M. Fauchet, "On-Chip Optical Interconnect Roadmap: Challenges and Critical Directions", IEEE Journal of Selected Topics in Quantum Electronics, Vol. 12, No. 6, pp1699-1705, Nov/Dec 2006

[9] G. Chen, Hui Chen, M. Maurylau, N.A. Nelson, D.H. Albonesi, P.M. Fauchet and E.G. Friedman, "On-Chip Copper-Based vs. Optical Interconnects: Delay Uncertainty, Latency, Power and Bandwidth Density Comparative Predictions", 2006 International Interconnect Technology Conference, pp 39-41, June 5, 2006.

[10] H. Ahmadi, W.E. Denzel, "A Survey of Modern High Performance Switching Technologies", IEEE Journal of Selected Areas in Communications, Vol. 7, No.7, pp.1091-1103, Sep 1989.

[11] Xiaohua Ma, G.S. Kuo,"Optical Switching Technology Comparison" Optical MEMS vs. other Technologies", IEEE Optical Communications, pp. S16-S23, Nov 2003.

[12] N.Andriollli, M. Scaffardi, A. Das Barman, P. Castoldi, L. Poti, A. Bogoni," All Optical Packet-Switched Interconnection Network Based on Modular Photonic Digital Processing", IET Communication, Vol.3, Issue.3, pp.477-486, 2009.

[13] G.Li, D.Huang, E. Yuceturk, P.J.Marchand, Sadik C. Esener, V.H.Ozguz, Y.Liu," Three-dimensional Optoelectronic Stacked Processor by use of Free-Space Optical Interconnection and Three-Dimensional VLSI Chip Stacks", Applied Optics, Vol. 41, No. 2, January 2002, pp.348-360.

[14] G.C.Marsden, P.J. Marchand, P.Harvey, Sadik C. Esener, "Optical Transpose Interconnection System Archnitectures", Optics Letters, Vol. 18. No. 13, July 1993, pp.1083- 1085.

[15] Guoqiang Li, E. Yuceturk,D. Huang, Sadik C. Esener,"Analysis of freespace optical interconnects for the three-dimensional optoelectronic stacked processor", Optics Communications, 202,2002, pp319-pp329.

[16] G.Marsden, P.J.Marchand,P.Harvey, Sadik C. Esener," Optical transpose interconnection system architectures", Optics Letters, Vol.18, No. 13, 1993, pp 1083-1085.

[17] O.Sj"olund,D. A. Louderback, E. R. Hegblom, J. Ko, and L. A. Coldren, "Monolithic Integration of Substrate Input/Output Resonant Photodetectors and Vertical-Cavity Lasers", IEEE Journal Of Quantum Electronics, Vol. 35, No.7,1999, pp1015-

[18] D.A. Louderback, O.Sjolund,E.R.Hegblom, J.Ko, L.A. Coldren, "FlipChip Bonded Arrays of Monolithically Integrated, Microlensed Vertical Cavity Lasers and Rosonant Photodetectos", IEEE Photonics Technology Letters, Vol. 11, No. 3, pp.304-pp306, March 1999.

[19] A. V. Krishnamoorthy, K. W. Goossen, L. M. F. Chirovsky, R. G. Rozier, P. Chandramani, W. S. Hobson, S. P. Hui, J. Lopata, J. A. Walker, and L. A. D'Asaro," 1616 VCSEL Array Flip-Chip Bonded to CMOS VLSI Circuit”, IEEE Photonics Technology Letters, Vol.12, NO. $8,2000$.

[20] S. Eitel, S. J. Fancey, H.-P. Gauggel, K.-H. Gulden, W. Bächtold, , and M. R. Taghizad," Highly Uniform Vertical-Cavity Surface-Emitting Lasers Integrated with Microlens Arrays", Vol. 12, No. 5, 2000.

[21] John A. Neff , "Thermal considerations of free-space optical interconnects using VCSEL-based smart pixel arrays", SPIE Proceeding Vol. 2691, pp 150-161,1996

[22] Y.C. Lee, Stanley E. Swirhun, W.S. Fu, T.A. Keyser, J.L. Jewell, W.E. Quinn, "Thermal Management of VCSEL Based Optoelectronic Modules",IEEE Transactions on Components,Packaging,and Manufacturing Technology, Part B, Vol. 19, 3, pp.549-547, 1996.

[23] T. Ouchi,"Thermal Analysis of thin-film Vertical Cavity Surface Emitting Lasers using Finite Element Method", Japan J. Appl. Phys, Vol.41, 2002, pp.5181-pp.5186

[24] D. Botez, D.R. Schifres, "Diode Laser Arrays", Chapter 4, "Modeling of Diode Laser Arrays", Cambridge University, Press.

[25] S.F. Yu, Analysis and Design of Vertical Cavity Surface Emitting Lasers, Wiley Interscience, Inc, Chapter 5, pp.193-pp.227.

[26] N. Bertra et al, "Modeling of Quantum Well lasers in electro-opto thermal interactions", IEEE Journal on Selected Topics in Quantum Electronic, Vol 1, No. 2, 1995, pp331-pp340. 